\title{
The Susceptibility to Antibiotics of Some Bacterial Strains Isolated from Cow Milk with Mastitis
}

\author{
George Cosmin NADǍŞ ${ }^{1}$, Nicodim FIȚ ${ }^{1}$, Cosmina BOUARI ${ }^{1}$, \\ Flore CHIRILÁ ${ }^{1}$, Sorin RĂPUNTEAN ${ }^{1}$, Vasile RUS ${ }^{1}$ \\ ${ }^{1}$ University of Agricultural Sciences and Veterinary Medicine, Faculty of Veterinary Medicine \\ 3-5 Mănăştur Street, 400372, Cluj-Napoca, Romania \\ * corresponding author e-mail: gnadas@usamvcluj.ro
}

Bulletin UASVM Veterinary Medicine 71(2) / 2014,

Print ISSN 1843-5270; Electronic ISSN 1843-5378

doi:10.15835/buasvmcn-vm: 10697

\begin{abstract}
Recent increased resistance to antibiotics of bacterial strains isolated from cow milk with mastitis represented a strong motivation for a study that would indicate the most efficient antibiotic for treatment. This study evaluated a total number of 31 samples collected from cows suffering from mastitis. Each sample was initially processed by the smear preparation method and Gram staining. Streaking methods were performed on both blood agar and glucose agar. The isolated pathogens were than tested regarding their sensitivity using Kirby Bauer disk diffusion test to the following antibiotics: Amoxicillin and clavulanic acid, Cefquinome, Enrofloxacin, Florfenicol, Gentamicin, Linco-spectin and Mastidiscs. The results demonstrated that single pathogens were involved in mastitis etiology in most of the cases, with rare associations. Staphylococci and streptococci strains were most frequently isolated, in 8 samples each, followed by E. coli with 4 strains and Klebsiella, Bacillus cereus and Corynebacterium in 1 sample. Regarding overall sensitivity, Amoxicillin and clavulanic acid was the most efficient antibiotic with an average of the inhibition area of $22.13 \mathrm{~mm}$, followed by Mastidiscs $(21.57 \mathrm{~mm})$, Enrofloxacin $(21.17 \mathrm{~mm})$, Linco-spectin (18.71 mm), Cefquinome (18.33), Florfenicol (17.76 mm) and Gentamicin (16.57). Our result demonstrates a good overall sensitivity of the tested strains.
\end{abstract}

Key words: antibiotic sensitivity, bacteria, cow milk, mastitis,

\section{INTRODUCTION}

Mastitis occurs in numerous species. Antimicrobial agents are used for treatment of infectious mastitis in dairy cattle, other livestock, companion animals, and humans. Mastitis is an economically important disease of dairy cattle and most mastitis research has focused on epidemiology and control of bovine mastitis. Antibiotic treatment of clinical and subclinical mastitis in dairy cattle is an established component of mastitis control programs (Barlow, 2011).

Recurrent mastitis in dairy cattle is a major concern of veterinarians and farmers, since the animal welfare, clinical status and production are impaired. Moreover the economic losses are huge since during the treatment and a few days after depending on the product, milk is forbidden to be used (3).

Most clinical mastitis (CM) cases of cows on dairy farms are treated with a standard intramammary antimicrobial treatment. Several antimicrobial treatments are available for $\mathrm{CM}$, differing in antimicrobial compound, route of application, duration, and cost. Because cow factors (e.g., parity, stage of lactation, and somatic cell count history) and the causal pathogen influence the probability of cure, cow-specific treatment of CM is often recommended (Steenveld et al., 2011).

Recent increased resistance to antibiotics of bacterial strains isolated from cow milk with mastitis represented a strong motivation for a study that aimed to indicate the most efficient antibiotic for treatment. 


\section{MATERIALS AND METHODS}

The investigations took place during October 2013 - May 2014 within the Microbiology Laboratory of the Faculty of Veterinary Medicine Cluj-Napoca. A total number of 31 milk samples were initially cultured on glucose agar and blood agar, and for each sample the identification of the bacterial specie involved in mastitis etiology was made by microscopic, cultural and biochemical examination. For each strain isolated, the test regarding its sensibility to antibiotics was realized using Kirby-Bauer disk diffusion test. The suspension from the tested strain at 0.5 tube of McFarland scale turbidity was dispersed on a plate with Mueller Hinton agar and the surface allowed drying. The antibiotics used were represented by Amoxicillin and clavulanic acid, Cefquinome, Enrofloxacin, Florfenicol, Gentamicin, Lincospectin and Mastidiscs.

\section{RESULTS AND DISCUSSION}

The results demonstrated that single pathogens were involved in mastitis etiology in most of the cases, with rare associations. Staphylococci and streptococci strains were most frequently isolated, in 8 samples each, followed by E. coli with 4 strains and Klebsiella, Bacillus cereus and Corynebacterium in 1 sample. Regarding overall sensitivity, Amoxicillin and clavulanic acid was the most efficient antibiotic with an average of the inhibition area of $22.13 \mathrm{~mm}$, followed by Mastidiscs (21.57 mm), Enrofloxacin $(21.17 \mathrm{~mm})$, Linco-spectin (18.71 mm), Cefquinome (18.33), Florfenicol (17.76 mm) and Gentamicin (16.57). Our result demonstrates a good overall sensitivity of the tested strains. The results regarding the sensitivity of the strains isolated from mastitis cow milk to antibiotics are presented in table 1 .
Continued use of antibiotics in the treatment and prevention of cow mastitis is a major subject of today's veterinary medicine. In order to prevent antibiotics to get into the humans and mostly children diet, it is required previous sensitivity testing and administration to be avoided as a preventive measure (McDougall et al., 2014). Control programs and herd management should also be considered. Moreover use of antibiotics in the treatment of mastitis in dairy cows for the last decades lead to some resistant strains (Oliver and Murinda, 2012).

Amoxicillin and clavulanic acid is an antibiotic administrated in many animal species, while for dairy cattle is available to be both administrated intramammary and systemic (intravenous). Enrofloxacin is a fluorochinolone antibiotic active against both Gram positive and Gram negative bacteria. Cefquinome is a fourth generation cephalosporin mostly available for the treatment of coliform mastitis.

The antibiotics evaluated within this study are frequently used in Transylvania for the treatment of mastitis. In the case of recurrent mastitis, usually the association of systemic and intramammary route is recommended, but only after the susceptibility testing. The most efficient antibiotic (Amoxicillin and clavulanic acid) is used for several decades but is still the one that scores the highest value of the average of diameter area.

\section{CONCLUSIONS}

The bacterial strains isolated from a total number of 31 samples of mastitis cow milk in Transylvania, are still sensitive to the most of the antibiotics evaluated.

Sensitivity testing using classic methods requires 48 hours for the determination, but the

Tab. 1. The sensitivity of bacterial strains to antibiotics

\begin{tabular}{cc}
\hline Antibiotic & $\begin{array}{c}\text { Average of the diameter } \\
\text { inhibition area } \mathbf{( m m}\end{array}$ \\
\hline Amoxicillin and clavulanic acid & 22.13 \\
\hline Mastidiscs & 21.57 \\
\hline Enrofloxacin & 21.17 \\
\hline Linco-spectin & 18.71 \\
\hline Cefquinome & 18.33 \\
\hline Florfenicol & 17.76 \\
\hline Gentamicin & 16.57
\end{tabular}


treatment is only recommended after the determination of susceptible antibiotic.

\section{REFERENCES}

1. Barlow J (2011). Mastitis therapy and antimicrobial susceptibility: a multispecies review with a focus on antibiotic treatment of mastitis in dairy cattle. J Mammary Gland Biol Neoplasia. 16(4):383-407.

2. McDougall S, Hussein H, Petrovski K (2014). Antimicrobial resistance in Staphylococcus aureus, Streptococcus uberis and Streptococcus dysgalactiae from dairy cows with mastitis. N Z Vet J. 62(2):68-76.

3. Oliveira L, Ruegg PL (2014). Treatments of clinical mastitis occurring in cows on 51 large dairy herds in Wisconsin. J Dairy Sci 97(9):5426-36.

4. Oliver SP, Murinda SE (2012). Antimicrobial resistance of mastitis pathogens. Vet Clin North Am Food Anim Pract.28(2):165-85.

5. Steeneveld W, van Werven T, Barkema HW, Hogeveen $\mathrm{H}$ (2011). Cow-specific treatment of clinical mastitis: an economic approach. J Dairy Sci 94(1):174-88. 